\title{
Rare cardiovascular diseases: from European legislations to classification and clinical practice
}

\author{
Piotr Podolec ${ }^{1}$, Jakub Stępniewski ${ }^{1}$, Jakub Podolec ${ }^{2}$, Grzegorz Kopeć ${ }^{1}$ \\ 'Department of Cardiac and Vascular Diseases, Institute of Cardiology, Jagiellonian University Medical College, John Paul II Hospital, \\ Centre for Rare Cardiovascular Diseases in Krakow, Poland \\ 2Department of Interventional Cardiology, Institute of Cardiology, Jagiellonian University Medical College, John Paul II Hospital, \\ Centre for Rare Cardiovascular Diseases in Krakow, Poland
}

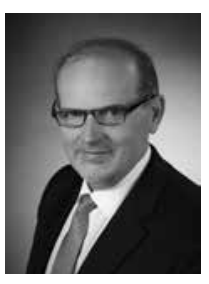

\section{DEFINITION AND EPIDEMIOLOGY OF RARE DISEASES}

Rare diseases (RD) are defined as life-threatening and chronically debilitating disorders with a prevalence of less than 5 per 10,000 of the general population [1]. It is estimated that up to $8 \%$ of European citizens may be affected by a RD [2]. Although these numbers seem small, they translate to around 27 to 36 million people suffering from RDs across Europe. According to the Orphanet database (http:// www.orpha.net), a designated reference portal for information on rare diseases and orphan drugs, there are 6,760 RD entities identified to date [3].

The definition of RDs, established by the European Union (EU) Parliament in 1999, reflects two fundamental aspects related to RDs. First, RDs are of such a low prevalence that accumulation of knowledge necessary to develop evidence-based management strategies requires multicentre networking. Second, due to a lack of appropriate knowledge and awareness about RDs in medical communities, these patients often hopelessly seek appropriate medical attention for extended periods of time, being continuously misdiagnosed or not diagnosed at all. The EurordisCare Survey Programme, investigating RDs patients' experiences and expectations regarding access to diagnosis and to health services, showed that as many as $40 \%$ of patients receive faulty diagnosis initially, which leads to execution of inappropriate medical interventions, including surgery, pharmacotherapy, or psychological treatment [4]. One-quarter of these people have to wait 5-30 years from the onset of symptoms to be accurately diagnosed, undergoing 4-5 consultations yearly. Additionally, around $25 \%$ of these patients need to travel to a different region to reach a diagnostic facility. The unmet needs of the growing number of patients with RDs in general call for special and combined efforts of regulatory authorities, health care providers, scientific committees, practitioners, and patient organisations.

\section{RARE CARDIOVASCULAR DISEASES. THE SCOPE OF THE PROBLEM}

The population of adults with RDs is widely heterogeneous with respect to type and complexity of diseases as well as their clinical manifestations, age of onset or natural history. They represent a subject of interest of almost all medical fields.

From all of the RDs registered in the Orphenet database a significant number have sole or, more commonly, concomitant cardiovascular manifestation [3]. The accurate number of rare cardiovascular diseases (RCD) is, however, difficult to assess due to the paucity of designated RCD registries and classifications, the complexity of these conditions, and their prevalence, which in many cases can be as low as $1<1,000,000$, as is the case in Loeys-Dietz syndrome, an aortic aneurysm syndrome due to transforming growth factor-beta receptor anomalies described in ten families, or in Alström syndrome, a multisystemic disorder characterised by dilated cardiomyopathy and cone-rod dystrophy, hearing loss, obesity, insulin resistance and hyperinsulinaemia, and progressive hepatic and renal dysfunction, which has been identified in about 450 patients worldwide [5]. Most of the RCDs have a genetic background [6]. Some of them are monogenic disorders, like Marfan or Brugada syndrome, but many have complex, multigenic causality. This includes development abnormalities in most of the congenital heart diseases or multifactorial origin of familial dilated cardiomyopathies. Other RCDs are the result of metabolic errors like Fabry disease, an alpha-galactosidase

\footnotetext{
Address for correspondence:

Piotr Podolec, MD, PhD, Department of Cardiac and Vascular Diseases, Institute of Cardiology, Jagiellonian University Medical College, John Paul II Hospital, Centre for Rare Cardiovascular Diseases in Krakow, ul. Prądnicka 80, 31-202 Kraków, Poland, e-mail: ppodolec@interia.pl Received: 29.09.2014 Accepted: 01.10.2014 Available as AoP: 28.10.2014

Copyright (C) Polskie Towarzystwo Kardiologiczne
} 
A deficiency syndrome or Gaucher disease, a glucocerebrosidase deficiency syndrome, autoimmune abnormalities like systemic vasculitis or connective tissue diseases affecting the heart, infectious like Lyme carditis or HIV-induced pulmonary arterial hypertension or neoplastic including primary or secondary cardiac tumours, as well as cardiovascular complications of non-cardiac tumours like secondary hypertension in the course of pheochromocytoma. This implies that the diversity of RCDs should not only be understood by the vast number of the diseases per se. The presence of rare combinations of more common conditions in one patient or uncommon phenotypes of cardiovascular disorders not necessarily of low prevalence also define the heterogeneity of RCDs and drive the need for attentive mulidisciplinarity in attending these patients.

The lack of disease specific therapy for most of the RCDs represents a substantial drawback in managing this group of patients. To date there are only a few medicinal products registered in the European Medicinal Agency for the treatment of orphan cardiovascular diseases [7]. These include a group of pulmonary arterial hypertension-specific drugs and enzyme replacement agents used to treat metabolic syndromes like Fabry disease, Hunter syndrome, Pompe or Gaucher disease, or mucopolysaccharidosis. Additionally, a number of RCDs may require an interventional approach, with development and improvement may required engaging efforts of highly specialised centres of various fields.

\section{RARE DISEASES: EU PRIORITY ACTION}

Recognising the significant disproportion in the access to high-quality healthcare, between patients with rare and common diseases, the EU authorities developed legislative acts such as Commission Communication Rare Diseases: Europe's Challenge (2008) [2] and Council Recommendation on an Action in the Field of Rare Diseases (2009) [8], in which they identified RDs as a priority for EU public health. Strategies presented in these texts concentrate on three main fields: (1) Improving Recognition and Visibility on Rare Diseases, (2) Supporting Policies on Rare Diseases in the Member States, (3) Developing European cooperation, coordination, and regulation for rare diseases. In order to apply these measures systematically, a coherent pan-European strategy was launched: the European Project for Rare Diseases National Plans Development (EUROPLAN). All countries have been advised to undertake efforts to elaborate public health plans aimed at the improvement of the quality of care for this population by implementing Council recommendations [9]. Following these guidelines, the Polish Ministry of Health has instituted a group of experts in the field of RDs, who elaborated the National Plan for RDs [10]. The plan concentrates on the formulation of classification and registries of RDs in Poland, creation of the reference centres networks, improvement in the access to highly specialised, integrated health care, and development of research and education in this field.

\section{RECOGNITION OF RARE DISEASES}

Raising awareness is the principal instrument to encourage recognition and tackle negligence of the issue of RDs [11]. Certain action points have been adopted into European legislation for this purpose. These include promotion of the universal RD definition, development of applicable codification and classification(s) systems, induction of research, and dissemination of accurate knowledge and information in a format adapted to the needs of professionals and patients. For these purposes an official reference portal has been established: the Orphanet.

\section{EUROPEAN CENTRES OF EXPERTISE}

In the field of RDs, where the expertise is scattered throughout Europe, designation of Centres of Expertise (CoE) or Centres of Reference is of particular importance. It has been underscored that no patient should feel neglected because he or she cannot have an access to a well-known, highly specialised centre composed of qualified individuals located nearby. For this reason, from 2004 onward, the Commission of High-Level Group on Health Services and Medical Care supported by the Rare Diseases Task Force Group began to elaborate on the concept of $\mathrm{CoE}$ [12]. This Working Group on European Centres of Reference ascertained that the idea of $\mathrm{CoE}$ is of highly positive value, which will provide benefit to patients and health professionals. A CoE for a RD or a group of RDs would bring together a panel of multidisciplinary competences, organised in a structured manner in order to accumulate expertise and maximise limited resources. The identification of a CoE varies, however, across Europe [13]. Some countries have expert clinics specialised by a disease or a group of diseases, and others have centres dedicated to the management of the majority of RDs in general. Some national centres are uniquely focused on the clinical management of patients, whilst others undertake clinical research only. This heterogeneity reflects the differences of national health care systems. It also represents the diversity of this group of diseases and the variability in the expertise and interests of involved physicians, experts, or researchers, and, for most, the breadth of the patients' needs and expectations. Being aware of this diversity on one hand, but recognising the need for unified definition of the concept of $\mathrm{CoE}$ on the other, the aforementioned Working Group followed by the labours of the European Union Committee of Experts on Rare Diseases (EUCERD) proposed a versatile model for establishing European $\mathrm{CoE}$, reflecting their mission, form, and scope of activities, criteria for designation and evaluation, and their European dimension $[14,15]$. CoE should therefore be able to provide high-quality healthcare services based on good practice guidelines, collaborate with legal authorities to establish public health strategies, cooperate with industry and patients' organisations, reinforce medical training and research to produce and disseminate evidence based knowledge, and 
be ready to form international co-operations. Adopting these recommendations in selecting $\mathrm{CoEs}$ at the national level is aimed at facilitating their recognition within patients' communities and local professionals, in order to improve the referral and diagnostic process. Another issue that these regulations highlight is the multi-disciplinary approach that the CoEs should demonstrate. The complexity and diversity of many RDs implies the need for integration of medical, paramedical, psychological, and social resources to provide service that cannot be delivered elsewhere with the same quality.

To date there are around $56 \mathrm{CoEs}$ focused on various types of RDs registered in Poland, of which at least three are identified as designated CoEs according to the Orphanet database (Table 1). The Centre for Rare Cardiovascular Diseases (CRCD) in the John Paul II Hospital in Krakow is regarded as a designated $\mathrm{CoE}$ for the diagnosis and treatment of RCDs [16]. The mission of the Centre is to (1) provide efficient diagnostic and therapeutic opportunities for RCD patients; (2) develop national and international collaboration networks; (3) disseminate knowledge, targeting both physicians and patients; (4) promote research and development; and (5) organise psychological and social support to patients and families (Table 2).

\section{EUROPEAN REFERENCE NETWORKS}

Given the specific nature of the matter of RDs, it becomes obvious however, that establishing CoEs with the highest expected expertise for each RD or group of RDs in each European country is an unrealistic concept. Therefore, creating networks of CoEs at the pan-European level appears to be the key element to achieve overall progress in this area [17]. As for the principle of the Cross-border Healthcare Directive, patients who cannot receive sufficient attention in their local health care facilities should have an opportunity to seek more adequate care in other CoEs hosted by other European Member States. But having to attend a clinic located in a foreign country can induce negative logistic, financial, or psychological outcomes for patients with often very debilitating rare conditions. The creation of the European Reference Networks (ERN) would, for this matter, ensure that the expertise would be the one that travels rather than the patients themselves. The current approach to the concept of ERNs is presented in the Recommendations on Rare Disease European Reference Networks document formulated by the EUCERD in 2013 [18]. Major potential benefits of ERNs are seen in (1) improving patient's access to multidisciplinary teams of experts by rearranging their referral pathways initiated by primary care physicians; (2) creating platforms for sharing knowledge and expertise, organising trainings for health professionals, and exchanging laboratory samples, radiological images, or diagnostic procedures; (3) establishing registries, databases, and biobanks to increase cohorts for research studies and clinical trials; and (4) bringing together patients and patient organisation networks to promote their impact in the field.

The CRCD in Krakow represents a node of the orphan cardiovascular diseases ERN [16]. It actively cooperates with a broad panel of national and international experts of various disciplines of medicine and health sciences (Table 2). The Centre has additionally elaborated referral pathways within district hospitals in the Malopolska region to improve access to medical advice for RCD patients and their physicians.

\section{RESEARCH AND DEVELOPMENT}

Amplifying research and development activities in the field of RDs requires specific initiatives to attract interest from researchers and industry. Academic research faces considerable disadvantages including lack of necessary collaborative efforts, fewer funding opportunities, the need for an alternative design for clinical trials, and a limited number of patients, which implies less interest for initiating clinical studies. For industry at the same time, RDs represent a small, niche market, which translates into expected low societal impact, and thus low profit. Nevertheless, the need to extend knowledge about epidemiology, pathophysiology or natural history of RDs and developing preventive, diagnostic and therapeutic interventions is unquestionable. This issue has been recognised by the Council Recommendations and is supported by several global initiatives. At the local and transnational level, research on RDs is being addressed as one of the priority areas in the health field under the EU Framework Programmes for Research and Technological Development (FP), which together with E-RARE the ERA-Net for Research Programmes on Rare Diseases and The International Rare Disease Research Consortium provide funding for over 4,690 ongoing research projects on about 2,177 different RDs [19]. Many of these programmes are recognised as disease registries. There are around 600 disease registries running in Europe according to the Orphanet database [20].

The CRCD in Krakow, in addition to serving as a platform for the exchange of expertise is intentionally engaged in the propagation of knowledge and research development in the field of RCDs (Table 2). A number of scientific initiatives are continuously being conducted in the Centre [21-27]. These include RCD registries that originate from a large database of patients with a variety of rare and non-rare cardiovascular diseases referred to the departments of the John Paul II Hospital in Krakow.

An issue requesting separate attention in the European legislation is the development and marketing of drugs to treat, prevent or diagnose rare conditions. Although the European Regulation on Orphan Medicinal Products has established a centralised procedure for designation of these products and has put in place incentives for the research, marketing and development, access to these medicines is often difficult and unequal from country to country [28]. According to analysis 
Table 1. List of Expert Centres in Poland identified by Orphanet*

\begin{tabular}{|c|c|}
\hline & Institution \\
\hline Cystic fibrosis clinic & Bialystok (Medical University) \\
\hline Rare inflammatory bowel disease & Torun (The Nicolaus Copernicus Medical University) \\
\hline Primary immunodeficiency clinic & Torun (The Nicolaus Copernicus Medical University) \\
\hline Primary immunodeficiency clinic & Gdansk (Medical University Clinical Centre) \\
\hline Rare systemic or rheumatologic disease clinic & Krakow (Jagiellonian University Medical College) \\
\hline Hereditary Angioedema Clinic ${ }^{R D}$ & Krakow (Clinical and Environmental Allergology, Jagiellonian University Medical College) \\
\hline Primary immunodeficiency clinic & Krakow (Polish-American Institute of Paediatrics, Jagiellonian University Medical College) \\
\hline Rare diabetes mellitus and Gaucher disease & Krakow (Polish-American Institute of Paediatrics, Jagiellonian University Medical College) \\
\hline Centre for Rare Cardiovascular Diseases ${ }^{\mathrm{RD}}$ & Krakow (John Paul II Hospital, Jagiellonian University Medical College) \\
\hline Cystic fibrosis and rare respiratory diseases & Rabka-Zdroj (National Institute for Tuberculosis and Lung Diseases) \\
\hline Primary immunodeficiency clinic & Lodz (Medical University) \\
\hline Rare pancreas and liver disease clinic & Lublin (Medical University) \\
\hline Rare paediatric cancers and haematological disorders & Lublin (Medical University) \\
\hline Primary immunodeficiency clinic & Poznan (Medical University) \\
\hline Congenital heart malformations clinic & Poznan (Medical University) \\
\hline Cystic fibrosis clinic & Poznan (Medical University) \\
\hline Rare digestive disease clinic & Szczecin (Pomeranian Medical University) \\
\hline Cystic fibrosis clinic & Karpacz (Pulmonology and Allergology Centre) \\
\hline Lymphoma and myeloid haemopathy oncologic clinic & Warsaw (Institute of Haematology and Transfusiology) \\
\hline Disorders of Haemostasis and Internal Medicine & Warsaw (Institute of Haematology and Transfusiology) \\
\hline General and Haematological Surgery Clinic & Warsaw (Institute of Haematology and Transfusiology) \\
\hline Cystic fibrosis clinic & Warsaw (Institute of Mother and Child) \\
\hline Clinic for rare pulmonary disease & Warsaw (Institute for Tuberculosis and Pulmonary Diseases) \\
\hline Porphyria clinic & Warsaw (Institute of Haematology and Transfusiology) \\
\hline Wilson Disease Clinic ${ }^{R D}$ & Warsaw (Institute of Psychiatry and Neurology) \\
\hline Paediatric rheumatologic clinic & Warsaw (Institute of Rheumatology) \\
\hline Rare breast cancer clinic & Warsaw (Maria Sklodowska-Curie Memorial Cancer, Centre and Institute of Oncology) \\
\hline Colorectal cancer clinic & Warsaw (Maria Sklodowska-Curie Memorial Cancer, Centre and Institute of Oncology) \\
\hline Rare gastrointestinal tumour clinic & Warsaw (Maria Sklodowska-Curie Memorial Cancer, Centre and Institute of Oncology) \\
\hline Gynaecological oncology clinic & Warsaw (Maria Sklodowska-Curie Memorial Cancer, Centre and Institute of Oncology) \\
\hline Rare lung cancer clinic & Warsaw (Maria Sklodowska-Curie Memorial Cancer, Centre and Institute of Oncology) \\
\hline Lymphoma clinic & Warsaw (Maria Sklodowska-Curie Memorial Cancer, Centre and Institute of Oncology) \\
\hline Neuro-oncology clinic & Warsaw (Maria Sklodowska-Curie Memorial Cancer, Centre and Institute of Oncology) \\
\hline Sarcoma clinic & Warsaw (Maria Sklodowska-Curie Memorial Cancer, Centre and Institute of Oncology) \\
\hline Urogenital cancer clinic & Warsaw (Maria Sklodowska-Curie Memorial Cancer, Centre and Institute of Oncology) \\
\hline Paediatric haematology clinic & Warsaw (Paediatric Hospital) \\
\hline Paediatric pneumology and allergology & Warsaw (Paediatric Hospital) \\
\hline Reference centre for metabolic diseases & Warsaw (The Children's Memorial Health Institute) \\
\hline Clinic for rare nephrology diseases & Warsaw (The Children's Memorial Health Institute) \\
\hline Clinic for rare hepatobiliary diseases & Warsaw (The Children's Memorial Health Institute) \\
\hline Cystic fibrosis clinic & Warsaw (The Children's Memorial Health Institute) \\
\hline Gastrology and hepatology clinic & Warsaw (The Children's Memorial Health Institute) \\
\hline Clinic for immunologic diseases & Warsaw (The Children's Memorial Health Institute) \\
\hline Clinic for rare surgical pediatric cardiac diseases & Warsaw (The Children's Memorial Health Institute) \\
\hline Clinic for rare surgical neurology diseases & Warsaw (The Children's Memorial Health Institute) \\
\hline Clinic for rare neurology diseases & Warsaw (The Children's Memorial Health Institute) \\
\hline Clinic for rare ophthalmology diseases & Warsaw (The Children's Memorial Health Institute) \\
\hline Clinic for oncologic diseases & Warsaw (The Children's Memorial Health Institute) \\
\hline Clinic for rare urology diseases & Warsaw (The Children's Memorial Health Institute) \\
\hline Congenital malformation surgical clinic & Warsaw (Medical University) \\
\hline Hirschprung disease clinic & Warsaw (Medical University) \\
\hline Rare neurologic disease clinic & Warsaw (Medical University) \\
\hline Epidemolysis Bullosa Clinic & Warsaw (Medical University) \\
\hline Primary immunodeficiency clinic & Wroclaw (Provincial Hospital) \\
\hline
\end{tabular}

$\mathrm{RD}$ - designated centres of expertise; *www.orpha.net 
Table 2. Activities of the Centre for Rare Cardiovascular Diseases (CRCD) in Krakow

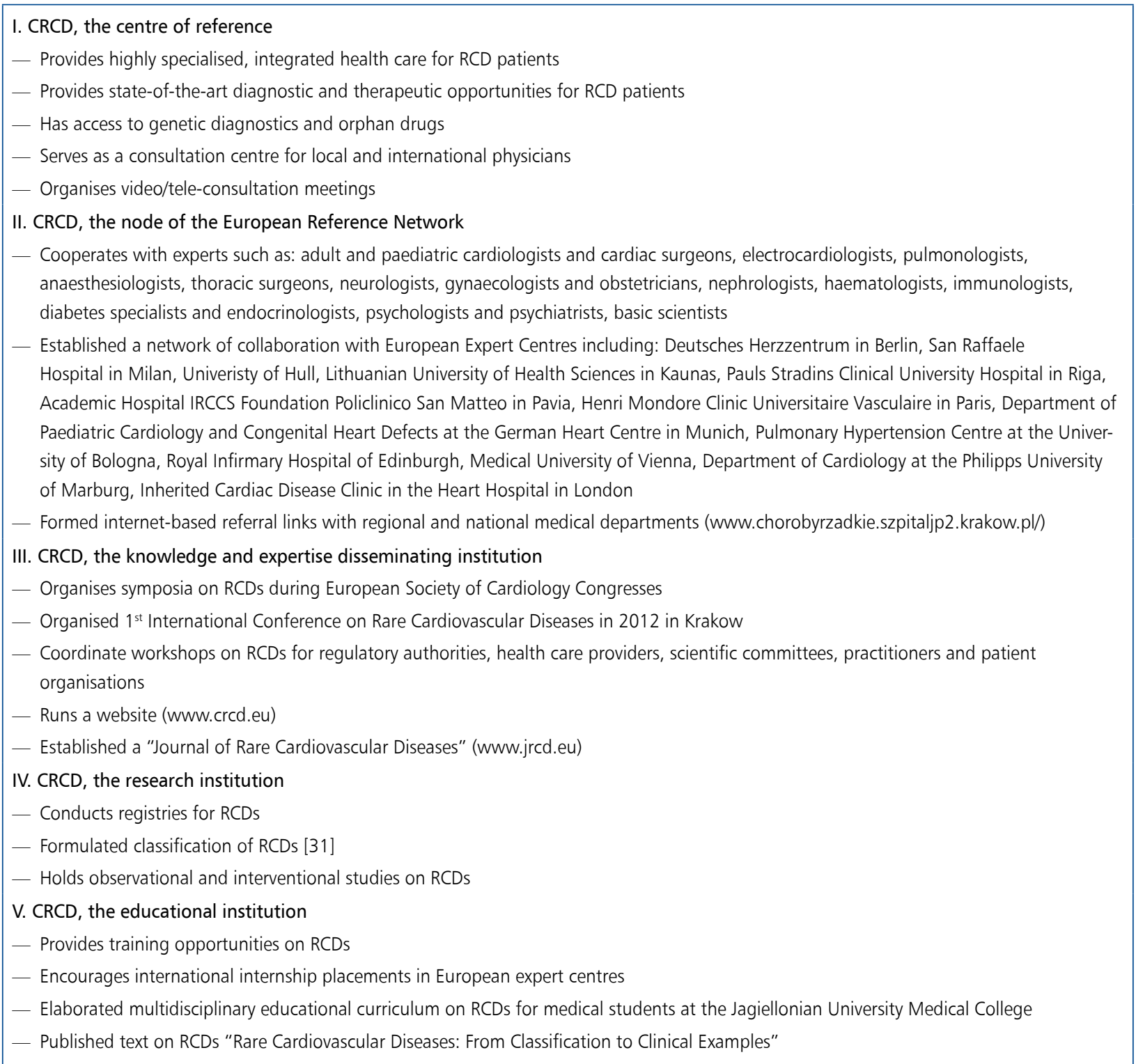

$\mathrm{RCD}$ - rare cardiovascular disease

carried out by Orphanet there are about 144 medicinal products authorised by the European market to potentially treat 120 diseases [29]. Development of novel therapeutics and elaboration of standards for clinical trials adequate to limited cohorts of patients is essential to achieve progress in this area [30].

\section{CLASSIFICATION OF RARE CARDIOVASCULAR DISEASES}

The lack of appropriate inventory of RDs remain an obstacle in promoting their recognition. Of the thousands of known RDs only about 250 have a distinct code in the existing $10^{\text {th }}$ version of the International Classification of Diseases (ICD). As a result, development of public health measures including prevalence, incidence, mortality, and morbidity is imposed. Disorders that are not visible to the health information systems consequently lack allocation of funds and resources. This creates a vicious cycle, which maintains the state of inequality. A few attempts is being undertaken to classify RDs. The Orphanet classification system, which is currently being developed, or the $11^{\text {th }}$ version of the ICD that is being revised by the World Health Organisation. Independently of the abovementioned projects the CRCD in Krakow has proposed an exhaustive classification of RCDs in order to support formulation of a common language in this field [31]. The RCD Classification derives from the experience of the Centre and a profound review of the majority of publications available through PubMed. It is aimed at facilitating recognition of RCDs and grouping the expertise in the main 
Table 3. Classification of Rare Cardiovascular Diseases

\begin{tabular}{|c|c|c|c|c|c|}
\hline Main Class & Group & Subgroup & Example & RCD code & ICD10 \\
\hline I - Rare diseases of systemic circulation & A...O & $1 \ldots 0$ & a...o & $\mathrm{I}-(\mathrm{A}) \cdot(1) \cdot(\mathrm{a})$ & $\ldots$ \\
\hline II - Rare diseases of pulmonary circulation & A...O & $1 \ldots 0$ & a...o & $\|-(A) \cdot(1) \cdot(a)$ & $\ldots$ \\
\hline III - Rare diseases of the heart (cardiomyopathies) & A...O & $1 \ldots 0$ & a...o & III-(A).(1).(a) & $\ldots$ \\
\hline IV - Rare congenital cardiovascular diseases & A...O & $1 \ldots 0$ & a...o & IV-(A).(1).(a) & $\ldots$ \\
\hline V-Rare arrhythmias & A...O & $1 \ldots 0$ & a...o & V-(A).(1).(a) & $\ldots$ \\
\hline $\mathrm{VI}$ - Cardiac tumours and cardiovascular diseases in malignancy & A...O & $1 \ldots 0$ & a...o & VI-(A).(1).(a) & $\ldots$ \\
\hline VII - Cardiovascular diseases in pregnancy & A...O & $1 \ldots 0$ & a...o & VII-(I)-(A).(1).(a) & $\ldots$ \\
\hline VIII — Unclassified rare cardiovascular diseases & A...O & $1 \ldots 0$ & a...o & VIII-(1) & $\ldots$ \\
\hline
\end{tabular}

Main classes of the classification accommodate the intensity of clinical symptoms and the underlining pathology. They are subdivided into groups and subgroups representing pathophysiological and symptomatological context. An appropriate example specifies the clinical setting. Each entity or group of entities is assigned a unique RCD code, which derives from the previously mentioned divisions. Example: I-1A.1 indicates a patient with anomaly of the circle of Willis, an entity included in class I of the RCD classification: I (class): rare diseases of the systemic circulation, 1 (group): anatomical malformations of the arteries, A (subgroup): cerebral arteries, 2 (example): anomalies of the circle of Willis. An attempt has also been made to refer to the existing $10^{\text {th }}$ version of the ICD system.

$\mathrm{RCD}$ - rare cardiovascular diseases; $\mathrm{O}$ - other

fields of RCDs. It provides a systemic inventory of RCDs based on the intensity of clinical symptoms and major pathological mechanisms. The main classes of the Classification include rare diseases of systemic circulation (Class I), rare diseases of pulmonary circulation (Class II), rare diseases of the heart (cardiomyopathies) (Class III), rare congenital cardiovascular diseases (Class IV), rare arrhythmias (Class V), cardiac tumours and cardiovascular diseases in malignancy (Class VI), cardiovascular diseases in pregnancy (Class VII), and unclassified rare cardiovascular diseases (Class VIII) (Table 3). They are further subdivided into groups and subgroups as appropriate. Each entity or group of entities is assigned unique RCD Classification code. An attempt has also been made to refer to the existing $10^{\text {th }}$ version of the ICD system by allocating its code; however, it was often not possible due to its evident limitations in terms of RCDs. The formulation of the RCD Classification was a challenging initiative and therefore it is currently undergoing an intensive review by international forum of experts.

\section{CONCLUSIONS}

RDs including RCDs are becoming a significant burden for patients, physicians and health care systems worldwide. RD patients often face overwhelming inequities in access to the professional care, which they would expect. Certain actions have been adopted at the European legislation level in order to overcome these barriers; however, a number of issues remain unresolved. Creation of the CoEs and ERNs concentrated on a particular RD or group of RDs, ignition of research and development, and facilitating the recognition are the key points in achieving progress in this field. The CRCD in the John Paul II Hospital in Krakow is an intentionally designed institution aimied at improving the care for RCDs patients. Being the national $\mathrm{CoE}$ and a part of the ERN, it serves as a forum for the exchange of knowledge and expertise supporting research and education in the field of orphan cardiovascular diseases. Its role in promoting awareness of RCDs has gained recognition among members of the national and international community of cardiologists after arranging symposia during the world's largest cardiology meetings, the European Society of Cardiology (ESC) Congresses in Paris (2011), Munich (2012), Amsterdam (2013), and Barcelona (2014) [32].

The RCD Classification, the "Journal of Rare Cardiovascular Diseases", the CRCD website, and the textbook Rare Cardiovascular Diseases: From Classification to Clinical Examples published by the Centre are tools in our commitment to establishing a routine dialogue among those who manage and care for patients who have so far been labelled as "no one's" or "orphan", a dialogue that include patient-specific discussions aimed at patient-specific solutions.

\section{Conflict of interest: none declared}

\section{References}

1. European Parliament. Decision No 1295/1999/EC of the European Parliament and of the Council of 29 April 1999 adopting a programme of Community action on rare diseases within the framework for action in the field of public health (1999 to 2003). Official Journal of the European Union, 1999; 42 (L155): 1-6.

2. European Commission. Communication from the Commission to the European Parliament, the Council, the European Economic and Social Committee and the Committee of the Regions on Rare diseases: Europe's challenges COM (2008) 679 final. [http://ec.europa.eu/health/ph_threats/non_com/docs/rare_ com_en.pdf].

3. Olry A. List of rare diseases and synonyms listed in alphabetical order. Orphanet Report Series, Rare Diseases Collection, 2014. http://www.orpha.net/orphacom/cahiers/docs/GB/List_of_ rare_diseases_in_alphabetical_order.pdf.

4. EURORDIS Report: The Voice of 12,000 Patients: Experiences \& Expectations of Rare Disease Patients on Diagnosis and Care in Europe. http://www.EURORDIS.org/article.php3?id_article=1960. 
5. Rath A. Prevalence of rare diseases: bibliographic data. Orphanet Report Series, Rare Diseases Collection, May 2014. http://www. orpha.net/orphacom/cahiers/docs/GB/Prevalence_of_rare_diseases_by_alphabetical_list.pdf.

6. Aymé S, Schmidtke J. Networking for rare diseases: a necessity for Europe. Bundesgesundheitsblatt Gesundheitsforschung Gesundheitsschutz, 2007; 50: 1477-83.

7. Rath A, Bécas-Garro MP. Lists of medicinal products for rare diseases in Europe. Orphanet Report Series, Orphan Drugs Collection, 2014. http://www.orpha.net/orphacom/cahiers/docs/GB/list_ of orphan drugs in europe.pdf.

8. European Council. Council Recommendation of 8 June 2009 on an action in the field of rare diseases (2009/C 151/02). Official Journal of the European Union, 2009; 52 (C151): 7-10.

9. European Parliament. Decision No 1786/2002/EC of the European Parliament and of the Council of 23 September 2002 adopting a programme of Community action in the field of public health (20032008). Official Journal of the European Union, 2002; 45 (L271): 1-14.

10. Ministerstwo Zdrowia. Zarządzenie Ministra Zdrowia z dnia 21 lipca 2008 roku, w sprawie powołania Zespołu do spraw Chorób Rzadkich. Dz.Urz.MZ.08.08.36.

11. European Commission. Commission Staff Working Document Accompanying the Communication from the Commission to the European Parliament, the Council, the European Economic and Social Committee and the Committee of the Regions on Rare diseases: Europe's challenges and the Proposal for a Council Recommendation on a European action in the field of rare diseases. Impact Assessment. 2012. http://eur-lex.europa.eu/legal-content/EN/TXT/PDF/?uri=CELEX:52012SC0212\&from=EN

12. European Commission. Report from the High Level Group to the Employment, Social Affairs, Health and Consumer Protection Council on 6-7 December 2004. http://ec.europa.eu/health/ph_overview/co operation/healthcare/docs/highlevel 2004026 en.pdf.

13. Aymé S, Rodwell Ch. The European Union Committee of Experts on Rare Diseases: three productive years at the service of the rare disease community. Orphanet J Rare Diseases, 2014; 9: 30

14. Rare Diseases Task Force: Centres of reference for rare diseases in Europe: State-of-the-art in 2006 and recommendations of the Rare Diseases Task Force. 2006 [http://ec.europa.eu/health/ph threats/non com/docs/contribution policy.pdf].

15. European Union Committee of Experts on Rare Diseases: Recommendations on quality criteria for centres of expertise for rare diseases in Member States. 2011 [http://www.eucerd. eu/?post_type $=$ document\&p=1224].

16. Podolec P. Rare Cardiovascular Diseases: From Classification to Clinical Examples. Centre for Rare Cardiovascular Diseases at the John Paul II Hospital in Krakow, Poland. Medycyna Praktyczna, Kraków 2013.

17. Aymé S. Rare diseases: a priority in public health and research J Rare Cardiovasc Dis. 2012; 1: 2-3.
18. European Union Committee of Experts on Rare Diseases: Recommendations on European Reference Networks for rare diseases. 2013 [http://www.eucerd.eu/?post_type=document\&p=2207].

19. Aymé S, Rodwell C eds. 2013 Report on the State of the Art of Rare Disease Activities in Europe, July 2013. http://www.eucerd. eu/upload/file/Reports/2013ReportStateofArtRDActivities.pdf.

20. Aymé S. Rare Disease Registries in Europe. Orphanet Report Series, Rare Diseases collection, January 2014. http://www.orpha. net/orphacom/cahiers/docs/GB/Registries.pdf.

21. Kopeć G, Moertl D, Miszalski-Jamka T et al. Left ventricular mass is preserved in patients with idiopathic pulmonary arterial hypertension and Eisenmenger's syndrome. Heart Lung Circ, 2014; 23: 454-461.

22. Kopeć G, Moertl D, Steiner S et al. Markers of thrombogenesis and fibrinolysis and their relation to inflammation and endothelial activation in patients with idiopathic pulmonary arterial hypertension. PLoS One, 2013; 8: e82628.

23. Kopeć G, Sobień B, Podolec M et al. The prevalence of abnormal echocardiographic findings in a sample of urban adult population. Kardiol Pol, 2014; 72: 42-49.

24. Kopeć G, Moertl D, Jankowski P et al. Pulmonary artery pulse wave velocity in idiopathic pulmonary arterial hypertension. Can J Cardiol, 2013; 29: 683-690.

25. Kopeć G, Tyrka A, Miszalski-Jamka T et al. Changes in exercise capacity and cardiac performance in a series of patients with Eisenmenger's syndrome transitioned from selective to dual endothelin receptor antagonist. Heart Lung Circ, 2012; 21: 671-678.

26. Kopeć G, Tyrka A, Miszalski-Jamka T et al. Electrocardiogram for the diagnosis of right ventricular hypertrophy and dilation in idiopathic pulmonary arterial hypertension. Circ J, 2012; 76: 1744-1749.

27. Tomkiewicz-Pajak L, Plazak W, Kolcz J et al. Iron deficiency and hematological changes in adult patients after Fontan operation. J Cardiol, 2014; 64: 384-389.

28. European Union Regulation on orphan medicinal products (regulation (EC) No 141/2000). [http://eur-lex.europa.eu/LexUriServ/LexUriServ.do?uri=OJ:L:2000:018:0001:0005:en:PDF].

29. Rath A, Bécas-Garro MP. Lists of medicinal products for rare diseases in Europe. Orphanet Report Series, Orphan Drugs Collection, July 2014. http://www.orpha.net/orphacom/cahiers/docs/GB/list_of_orphan_drugs_in_europe.pdf.

30. Cornu C, Kassai B, Fisch R et al. Experimental designs for small randomized clinical trials: an algorithm for choice. Orphanet J Rare Diseases, 2013; 8: 48.

31. Podolec P. Classification of Rare Cardiovascular Diseases (RCD Classification), Krakow 2013. J Rare Cardiovasc Dis, 2013; 2: 13-24.

32. Musialek P. 2nd Symposium on Rare Cardiovascular Diseases: ESC Munich 2012: Chairperson's Perspective 30. J Rare Cardiovasc Dis, 2012; 1: 30-31. 